\title{
Influence of Temperature on Characters of Thermoelectric Generators Based on Test Bed
}

\author{
Zongzheng Ma, Xinli Wang, and Anjie Yang \\ Department of Mechanical Engineering, Henan Institute of Engineering, Zhengzhou 451191, China \\ Correspondence should be addressed to Zongzheng Ma; zongzhengma@163.com
}

Received 5 January 2014; Accepted 31 March 2014; Published 13 April 2014

Academic Editor: Yu-Lun Chueh

Copyright (C) 2014 Zongzheng Ma et al. This is an open access article distributed under the Creative Commons Attribution License, which permits unrestricted use, distribution, and reproduction in any medium, provided the original work is properly cited.

In order to achieve the energy recovery of the coolant heat for internal combustion engine (ICE) using the thermoelectric generation (TEG) technology, one test bed for studying the influence of temperature on the characters of thermoelectric generators was established and the relationship between the temperature and characters of thermoelectric generator was researched based on it. The results showed that the cooling effect improved with the increase of fan speed which the fan was installed in the vertical direction of the radiator, but the cooling effect had a limit speed value. And it also indicated that the forced air cooling was better than the natural convection cooling method which can effectively reduce the temperature of the cold end while it has little effect on the hot end temperature. Moreover, the Seebeck coefficient was reduced with the increase of temperature difference between the two ends of thermoelectric generator and the Seebeck coefficient was also declined with one end temperature rise when the other end temperature was constant.

\section{Introduction}

Conventional internal combustion engine (ICE) thermal efficiency is only about $40 \%$ of the fuel energy, while about as much as 60 percent of the energy has not been effectively utilized which results in the fact that most of the energy is lost to the air in the form of heat and a huge waste of energy [1]. Therefore, if the fuel economy and engine thermal efficiency can be improved effetely it has an important significance for energy conservation and environment pollution.

Thermoelectric generation technology (TEG) is a new kind of energy recovery technology which can convert heat into electricity directly using the Seebeck effect of thermoelectric materials. Therefore, based on thermoelectric conversion principle the engine exhaust gas heat and coolant heat can be recovered. This kind of energy recovery technology has stable performance, no noise, no wear, small size, light weight, long life, and other advantages which have caught more and more attention in recent years $[2,3]$.

There are two types of engine energy that can be recycled using TEG technology; one is the coolant and the other is the exhaust gas. Currently, many researchers are paying more attention to the relationship of thermoelectric structure [4], cooling method [5-7], fin length [8], and radiator structure [9-11] on heat transfer efficiency for engine exhaust gas energy recovery. While few researches have been done about coolant energy recovery and only one explore by using thermoelectric power generation to replace the radiator has been reported whose results showed that the energy recovery efficiency was $3.2 \%$ and $10 \%$ when the speed was $80 \mathrm{~km} / \mathrm{h}$ and at idle speed respectively [12].

The main reason of fewer researches on coolant energy recovery is that the coolant temperature generally does not exceed 110 degrees centigrade which is much lower than the exhaust gas temperature. However, in the view of engine heat balance, coolant energy is also considerable compared with the engine exhaust gas energy. Meanwhile the cooling system has cooling fans and pumps at the present which provides good premise for TEG and the TEG modules are commercially available without using special production whose temperature limit is 220 degrees centigrade which is higher than the coolant temperature. So it is very convenient for practical application with the commercial TEG for coolant energy recovery.

For the application of TEG technology for coolant energy recovery, the first thing is to reveal the effect of temperature 


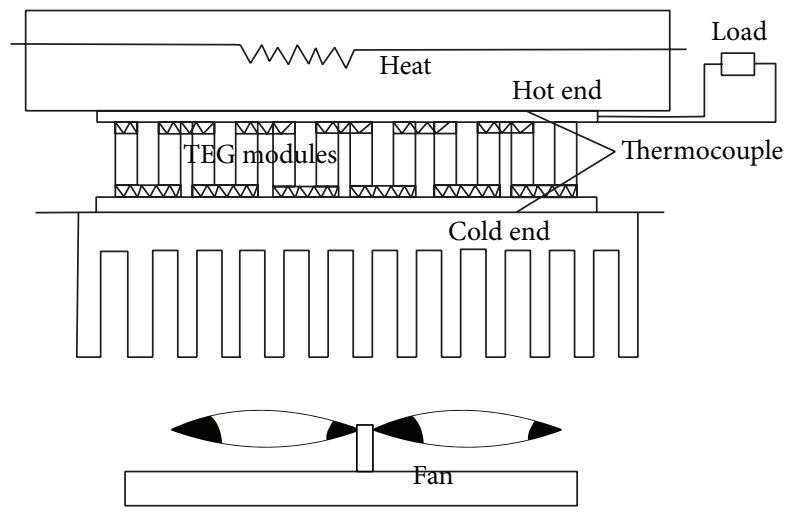

Figure 1: Diagram of TEG module test.

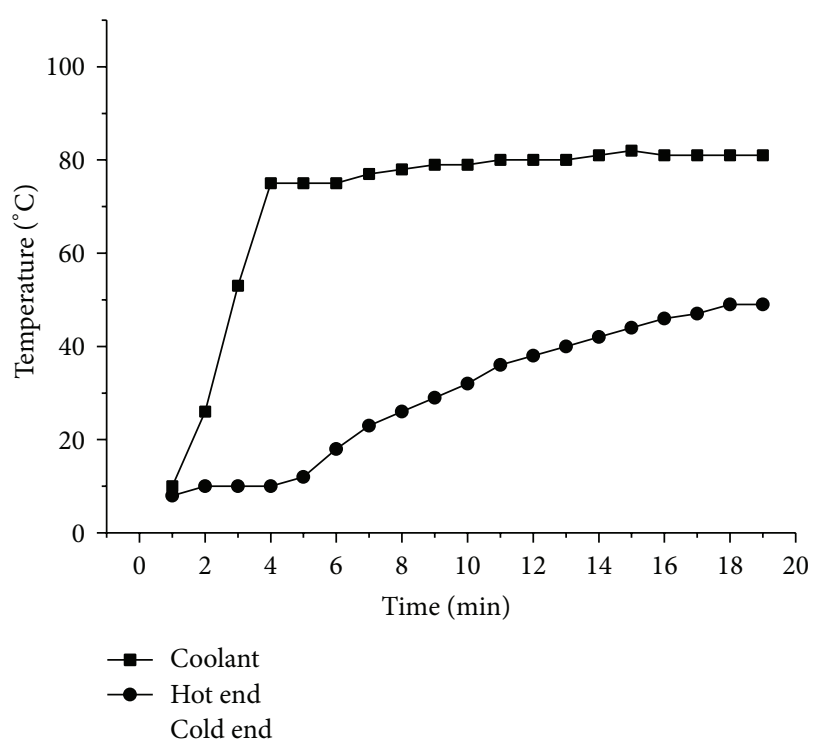

FIgURE 2: Temperature trend during the coolant temperature rise.

on the characters of the thermoelectric generator. So one test bed was established which can be used to study the characters of TEG based on analysis of engine coolant heat energy recovery.

\section{Test Bed Set}

The schematic diagram of the TEG test bed is shown in Figure 1 and the system can be divided into four parts: the cold end, the hot end, the TEG modules, and the load. Among them, the cold end is made of aluminum and the dimension $(L \times W \times H)$ is $300 \mathrm{~mm} \times 100 \mathrm{~mm} \times 35 \mathrm{~mm}$ while the thickness is $5 \mathrm{~mm}$ which is cooled with forced air. The hot end is made of cast aluminum and the shape is a box which can contain pure water for heating. The TEG model type is F30345 and size is $40 \times 40 \times 3.8 \mathrm{~mm}$ whose internal resistance is $2.4 \Omega$. In order to measure the temperature of the cold and hot side the flat type $K$ thermocouple is applied whose width is $3.7 \mathrm{~mm}$ and thickness is only $0.28 \mathrm{~mm}$ and the accuracy is \pm 1.5 degrees centigrade and the reaction time is $2.50 \mathrm{~s}$ which is suited for measurement requirements. For increasing thermal conductivity and contact of the cold and hot end the silicone is used to connect the four parts. Meanwhile, in order to measure the temperature of coolant and ambient, respectively, the $K$-type nickel-chromium- nickel silicon thermocouple is also used.

Then the processing circuit for thermocouple and Altai's USB2002 acquisition system are also used for data acquisition. At the end the real-time recording temperature, voltage, and other experimental data are gathered into a PC for data storage.

\section{Results and Discussion}

3.1. Process of the Temperature Rise. Figure 2 shows temperature trend of the hot end and cold end during the coolant temperature rise process. It can be seen that the hot end has a same trend as the coolant during the heating process, but at the end the coolant temperature reaches 100 degrees centigrade while the hot end temperature is only 80 degrees centigrade. The reason is the thermal resistance of the aluminum container wall between the coolant and the hot end, which causes the temperature difference between the coolant and the hot end. Therefore, in practical applications, this kind of thermal resistance should be minimized and the material with big thermal conductivity such as brass can be considered.

However, the temperature rise trend of the cold end shows a significantly different chat during the heating process. From the figure it can be seen that there is a $4 \mathrm{~s}$ delay of the cold end and the temperature of the cold end is almost the same as the ambient temperature at the first $4 \mathrm{~s}$ of the heating process. Then the temperature rises with the coolant temperature and the temperature keeps at 49 degrees centigrade at the end naming about $18 \mathrm{~s}$ later than the start.

The material of the cold and the hot end of TEG modules is ceramic and the core material is the semiconductor whose thermal conductivity is small. So at the beginning the cold end temperature remains the ambient temperature.

But, with the continuous heating process, the cold end temperature rises slowly. It can be explained by the small distance between the cold end and the TEG modules. Because of the small distance between the hot end and the cold end, 
the heat can be transmitted through the TEG modules and the surrounding air even though their thermal conductivity is small. However, when the heat transmitted to the hot end is equal to the heat transferred to the heat sink the temperature does not rise.

3.2. Effect of Cooling Methods on Temperature. From the above analysis, the temperature difference across the TEG can be kept at about 30 degrees centigrade using natural air cooling method. For generating more power larger temperature difference is needed so the forced air cooling method is applied. The fan used in this test is voltage control and rated voltage is $12 \mathrm{~V}$; therefore, several different cooling effects in the analysis are simulated by changing the voltage of the fan.

Figure 3 shows the temperature of both ends of TEG at different voltages. Compared with nature air cooling method the temperature difference across TEG is significantly reduced by approximately 24 degrees centigrade which can make the temperature difference reach 50 degrees centigrade or more. So it is obvious that forced air cooling can increase temperature difference. It also can be found that the hot side temperature is reduced by about 2 degrees centigrade although the cooling fan is located in the cold end. According to heat theory, there are three ways for heat transmission: heat conduction, convection, and radiation. For this test the heat conduction plays an important role in heat transmission and the hot end heat obtained from the coolant is constant. The cold end temperature decreases obviously which can improve the heat radiation and reduce the hot end temperature when the surrounding ambient temperature of TEG is reduced.

It also can be found in Figure 3 that the cold end temperature decreases become obvious when the fan voltage rises from $6 \mathrm{~V}$ to $12 \mathrm{~V}$. Namely, forced air cooling can improve the cooling effect, but there is a limit.

The cooling method adopted in this test is an axial fan plus radiator, namely, the jet cooling method whose cooling air flow direction is the vertical direction of the radiator which will cause pressure loss. When the fan speed continues to increase the pressure loss is also increasing. Then the cooling effect is getting smaller and smaller with the increase of fan speed [13].

3.3. Influence of Temperature Difference in Seebeck Coefficient. It is well known that the Seebeck coefficient can be defined as

$$
\alpha=\frac{V_{o}}{\left(T_{H}-T_{C}\right)},
$$

where $\alpha$ is Seebeck coefficient; $V_{O}$ is open circuit voltage, $\mathrm{V}$; $T_{H}$ is temperature of hot end, ${ }^{\circ} \mathrm{C} ; T_{C}$ is temperature of cool end, ${ }^{\circ} \mathrm{C}$.

Figure 4 shows the open circuit voltage increase with the temperature difference of both ends and the fitter line. It is revealed that the open circuit voltage has an almost linear relationship with the temperature difference of both ends, but it still has some error between the experiment points and linear fitter line which means the Seebeck coefficient is not constant. So the influence factor is researched in the following part.

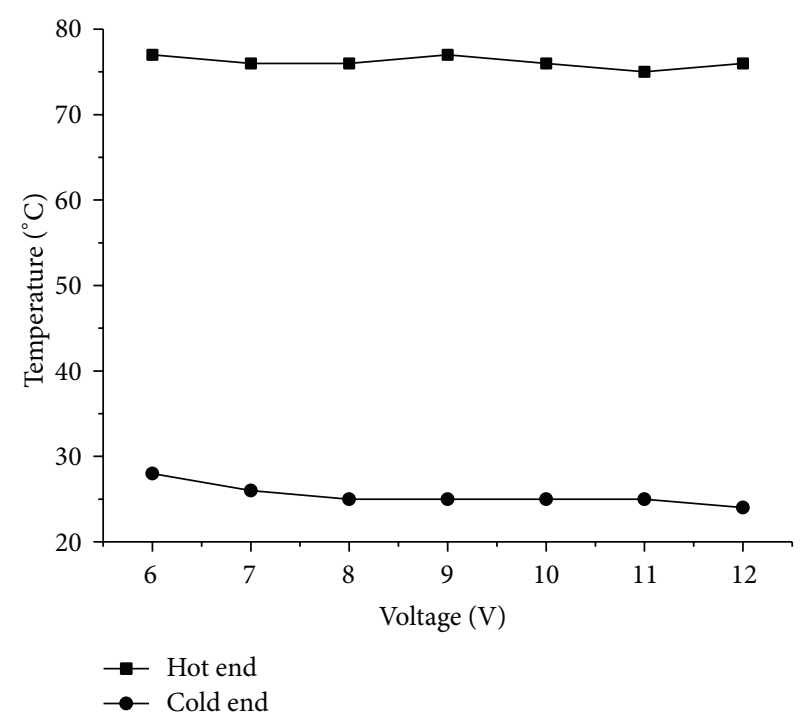

FIGURE 3: Temperature of both ends of TEG at different input voltages.

Figure 5 shows the curve between the temperature difference of both ends and the Seebeck coefficient when the data is measured during the temperature rise process and the Seebeck coefficient is calculated by (1). It is revealed that when the temperature difference across TEG ends is 16 degrees centigrade the Seebeck coefficient was 0.049 and the Seebeck coefficient value continue to decrease with the rise of temperature difference. And when the temperature difference is 65 degrees centigrade the Seebeck coefficient decreases to 0.031 . It can be concluded that the Seebeck coefficient decreases with the increase of temperature difference.

It can be found from the material of TEG that the $\mathrm{N}$-type material is mainly $\mathrm{Bi}_{2} \mathrm{Te}$ and P-type material is mainly $\mathrm{Sb}_{2} \mathrm{Te}_{3}$. It is known about the characteristics of semimonomer material that Seebeck coefficient is reduced with the increase of temperature. For this experiment both the hot and cold end temperature increase during the heating process, so the Seebeck coefficient of the TEG decreases with the temperature increase.

Note that, when the temperature difference increases the open circuit voltage is increased because of temperature difference increase although the Seebeck coefficient decreases. So the higher temperature difference across TEG higher open circuit voltage can be reached.

From the above analysis, it can be seen that the temperature difference has an effect on Seebeck coefficient, which also means the Seebeck coefficient is not constant. Therefore, for further analysis the temperature of the hot end and cold end is fixed constant, respectively.

3.4. The Effect of TEG Ends Temperature on Seebeck Coefficient. Figure 6 shows the Seebeck coefficient change with the cold end temperature with the hot end temperature being fixed at 80 degrees centigrade and the Seebeck coefficient being also calculated using (1). It can be seen that when the cold end temperature is 25 degrees centigrade the Seebeck coefficient is about 0.038 while the Seebeck coefficient is 


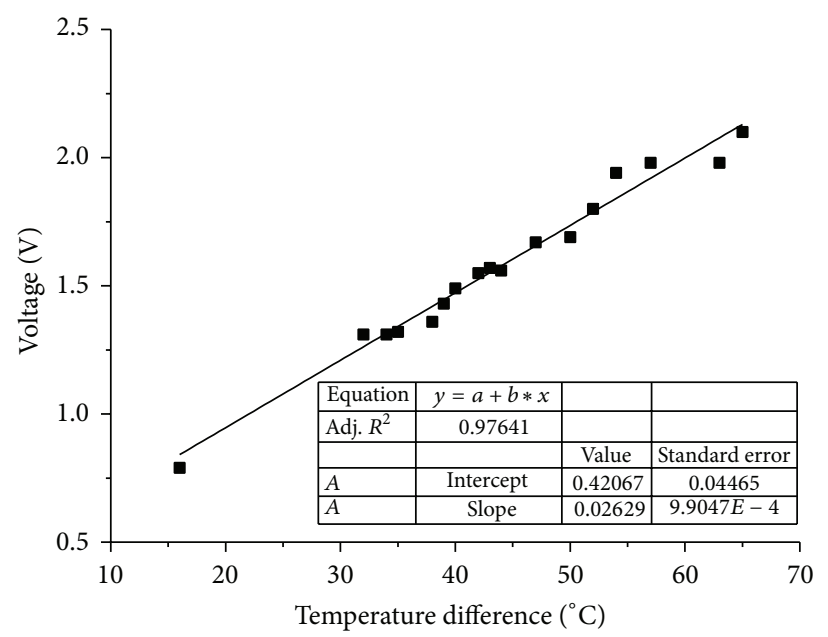

- Open circuit voltage

- Linear fit of $A$

FIGURE 4: Open circuit voltage increase with the temperature difference.

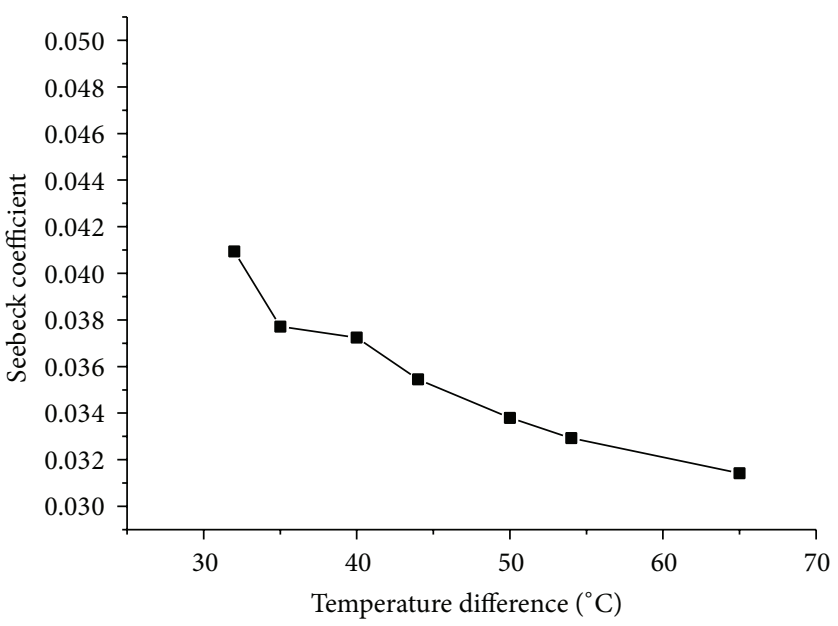

FIGURE 5: Seebeck coefficient of temperature difference.

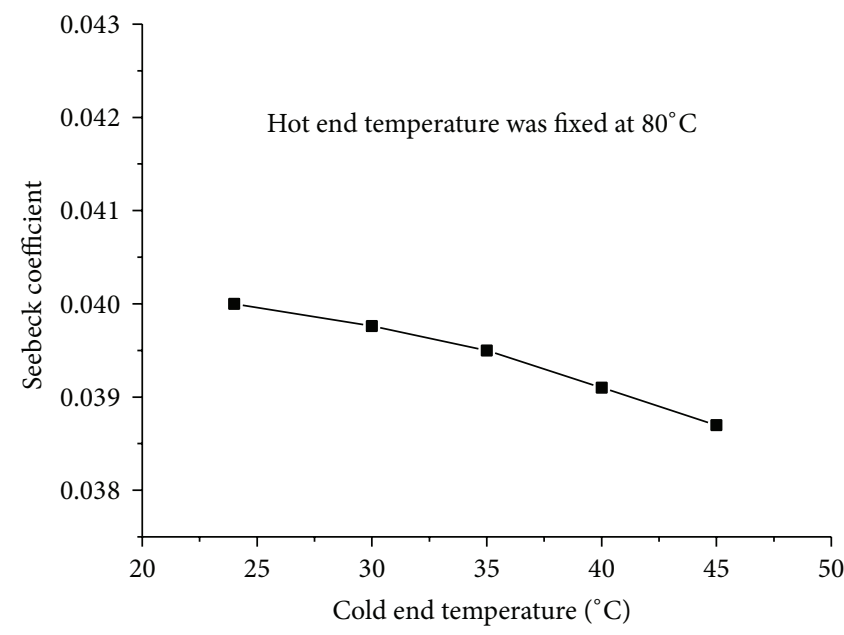

FIGURE 6: Seebeck coefficient of different cold end temperature. 


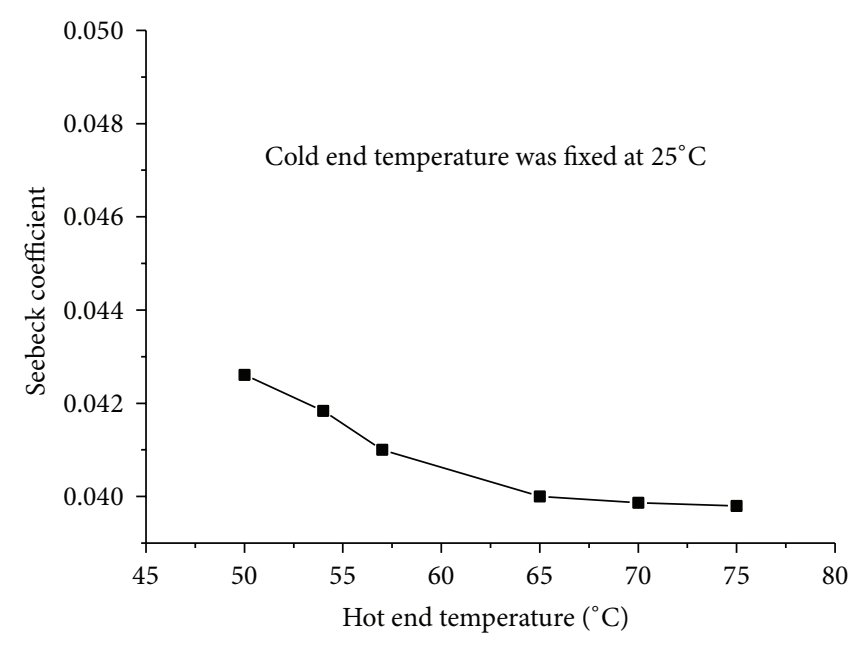

Figure 7: Seebeck coefficient of different hot end temperature.

only 0.002 which is decreased by $5 \%$ when the cold end temperature is 45 degrees centigrade. It is revealed that the Seebeck coefficient of TEG decreases with the cold end temperature rise and the hot end temperature fixed.

From the foregoing analysis, the Seebeck coefficient of the semiconductor material reduces with the temperature rise. So when the temperature of the hot end is fixed, the Seebeck coefficient reduces with cold end temperature rise, which also based on the characters of semiconductor material.

The same trend can be seen in Figure 7 which shows the Seebeck coefficient change with the hot end temperature with the cold end temperature being fixed at 25 degrees centigrade. It can be reached from the figure that when the hot end temperature is 50 degrees centigrade the Seebeck coefficient is about 0.042 , while the Seebeck coefficient is only 0.039 which is reduced by approximately 0.003 when the cold end temperature is 75 degrees centigrade.

\section{Conclusion}

Based on the test bed the relationship between the temperature and characters of TEG was studied and the following conclusions can be reached.

(1) The forced air cooling method can effectively reduce the temperature of the cold end and has little influence on the hot end of TEG and it is recommended for energy recovery based on TEG.

(2) Using the axial fan plus the radiator cooling method has a speed limit and the cooling effect is almost unchanged with the increase of rotational speed when the fan speed reaches the limit because of pressure lose.

(3) The Seebeck coefficient was reduced with the increase of the difference between the two ends of thermoelectric generator because of the characteristics of semimonomer material that the Seebeck coefficient is reduced with temperature increase.
(4) The Seebeck coefficient is also declined with one end temperature rise while the other end temperature is fixed and the reason is also the characteristics of semimonomer material.

\section{Conflict of Interests}

The authors declare that there is no conflict of interests regarding the publication of this paper.

\section{Acknowledgments}

This work is supported by Science and Technology Project of Henan Province (no. 132102210459), Foundation of Henan Educational Committee (13B480007), and Doctoral Fund of Henan Institute of Engineering (D2012011). The authors would like to express appreciation of financial support by the Power-driven Machinery and Vehicle Engineering Research Center and the Key Subject of Machine Design and Theory of Henan Institute of Engineering.

\section{References}

[1] H. Y. Liu, W. Z. Liu, and Q. He, "Study on remaining heat power generation in engine vent-pipe," Journal of Changchun University of Science and Technology: Natural Science, vol. 30, no. 3, pp. 70-72, 2007.

[2] G. J. Snyder and E. S. Toberer, "Complex thermoelectric materials," Nature Materials, vol. 7, no. 2, pp. 105-114, 2008.

[3] R. Y. Nuwayhid, A. Shihadeh, and N. Ghaddar, "Development and testing of a domestic woodstove thermoelectric generator with natural convection cooling," Energy Conversion and Management, vol. 46, no. 9-10, pp. 1631-1643, 2005.

[4] H. W. Liu and Z. Zh, "Research on a novel thermo-electric generator structure for vehicle exhaust gas energy recovery," Energy Conservation Technology, no. 6, pp. 507-509, 2006.

[5] E. Hatzikraniotis, "On the recovery of wasted heat using a commercial thermo-electric device," in Proceedings of the International Congress on Advances in Applied Physics and Materials Science, pp. 286-289, Antalya, Turkey, 2011. 
[6] J. Yu and H. Zhao, "A numerical model for thermoelectric generator with the parallel-plate heat exchanger," Journal of Power Sources, vol. 172, no. 1, pp. 428-434, 2007.

[7] M. D. Li, J. Qu, and Y. D. Li, "Influence of contact effects on properties of a small thermo-electric power generator," Chinese Journal of Semiconductors, vol. 26, no. 12, pp. 2440-2444, 2005.

[8] W. H. Chen, Y. L. Chen, I. H. Chen, and W.-L. Huang, "Experimental study on thermo-electric modules for power generation at various operating conditions," Energy, vol. 45, no. 1, pp. 874-881, 2012.

[9] Z.-G. Zhou, D.-S. Zhu, H.-X. Wu, and H.-S. Zhang, "Heat transfer characteristic analysis and experimental investigation of thermoelectric generator," Journal of South China University of Technology: Natural Science, vol. 39, no. 11, pp. 47-52, 2011.

[10] G. Casano and S. Piva, "Experimental investigation of the performance of a thermoelectric generator based on Peltier cells," Experimental Thermal and Fluid Science, vol. 35, no. 4, pp. 660669, 2011.

[11] D. Dai, Y. Zhou, and J. Liu, "Liquid metal based thermoelectric generation system for waste heat recovery," Renewable Energy, vol. 36, no. 12, pp. 3530-3536, 2011.

[12] R. Quan, S.-H. Quan, L. Huang, Y.-D. Deng, and X.-F. Tang, "Design and initial performance experiments of thermoelectric generator based on exhaust heat of car," Journal of Shanghai Jiaotong University, vol. 45, no. 6, pp. 842-850, 2011.

[13] W. B. Zheng, Y. Wang, and Z. F. Wu, "Testing platform for the thermo-electric properties of thermo-electric generators," Experimental Technology and Management, vol. 23, no. 11, pp. 62-65, 2006. 

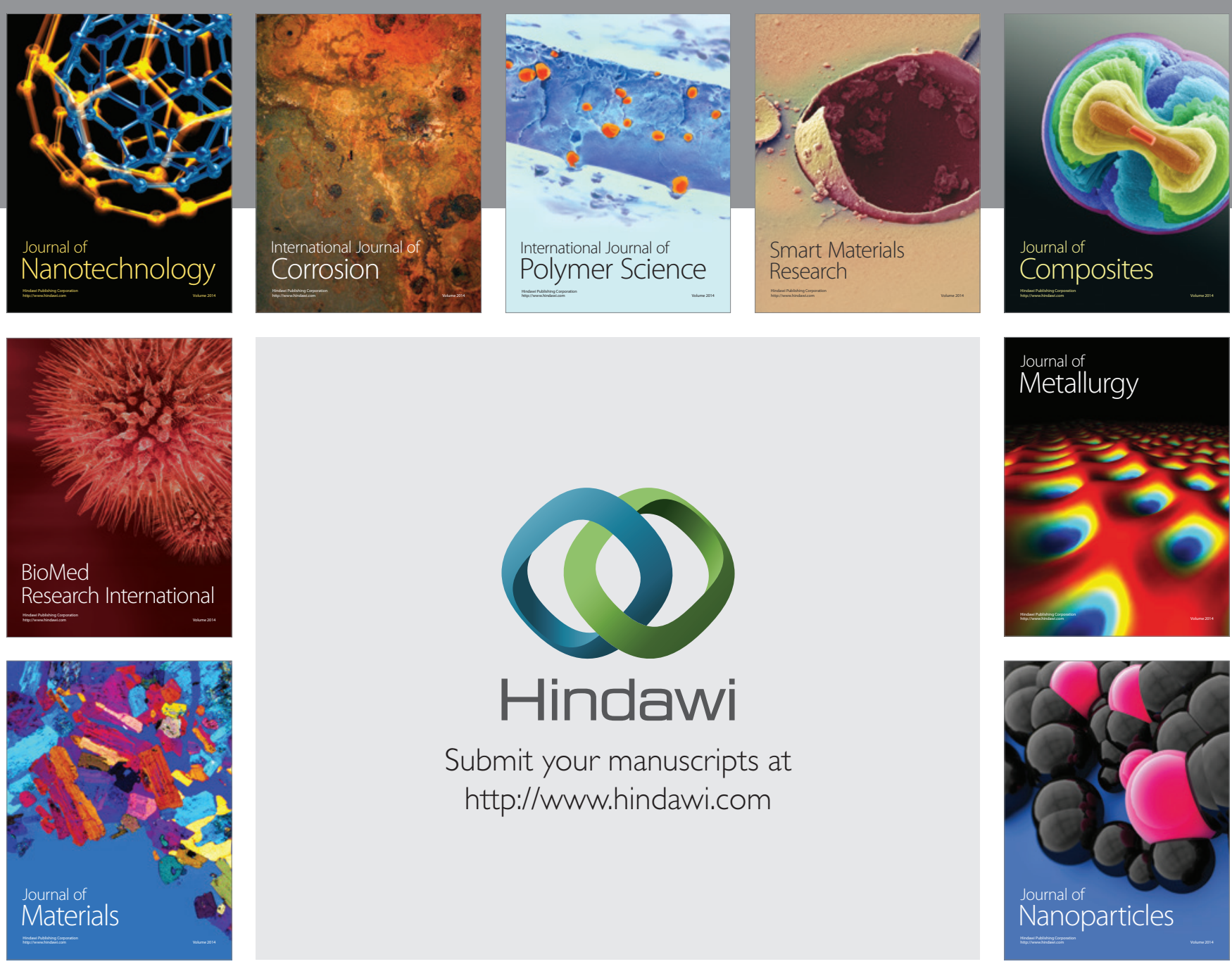

Submit your manuscripts at http://www.hindawi.com
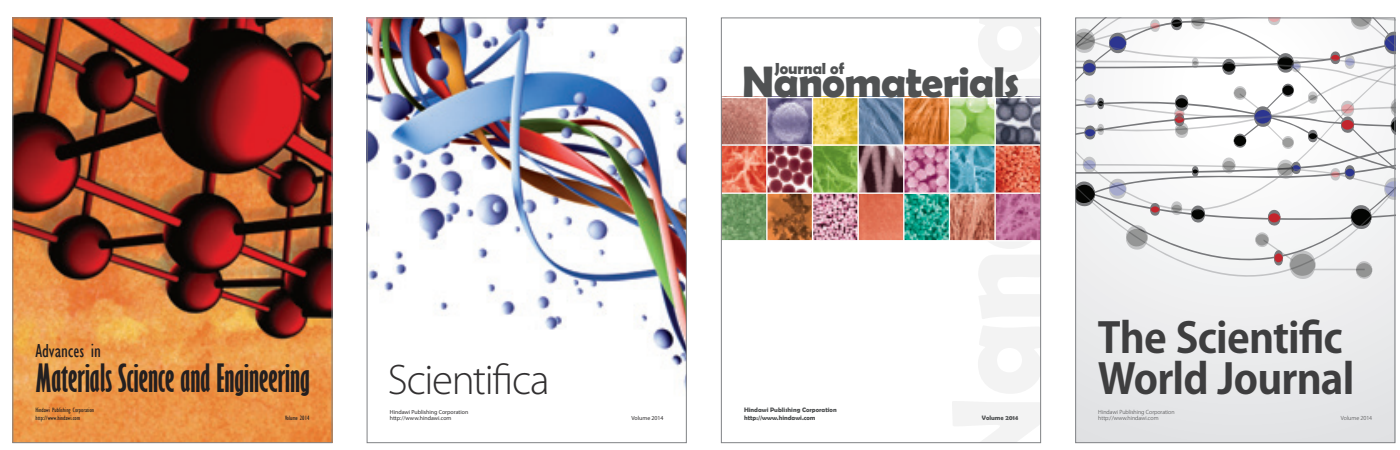

\section{The Scientific World Journal}
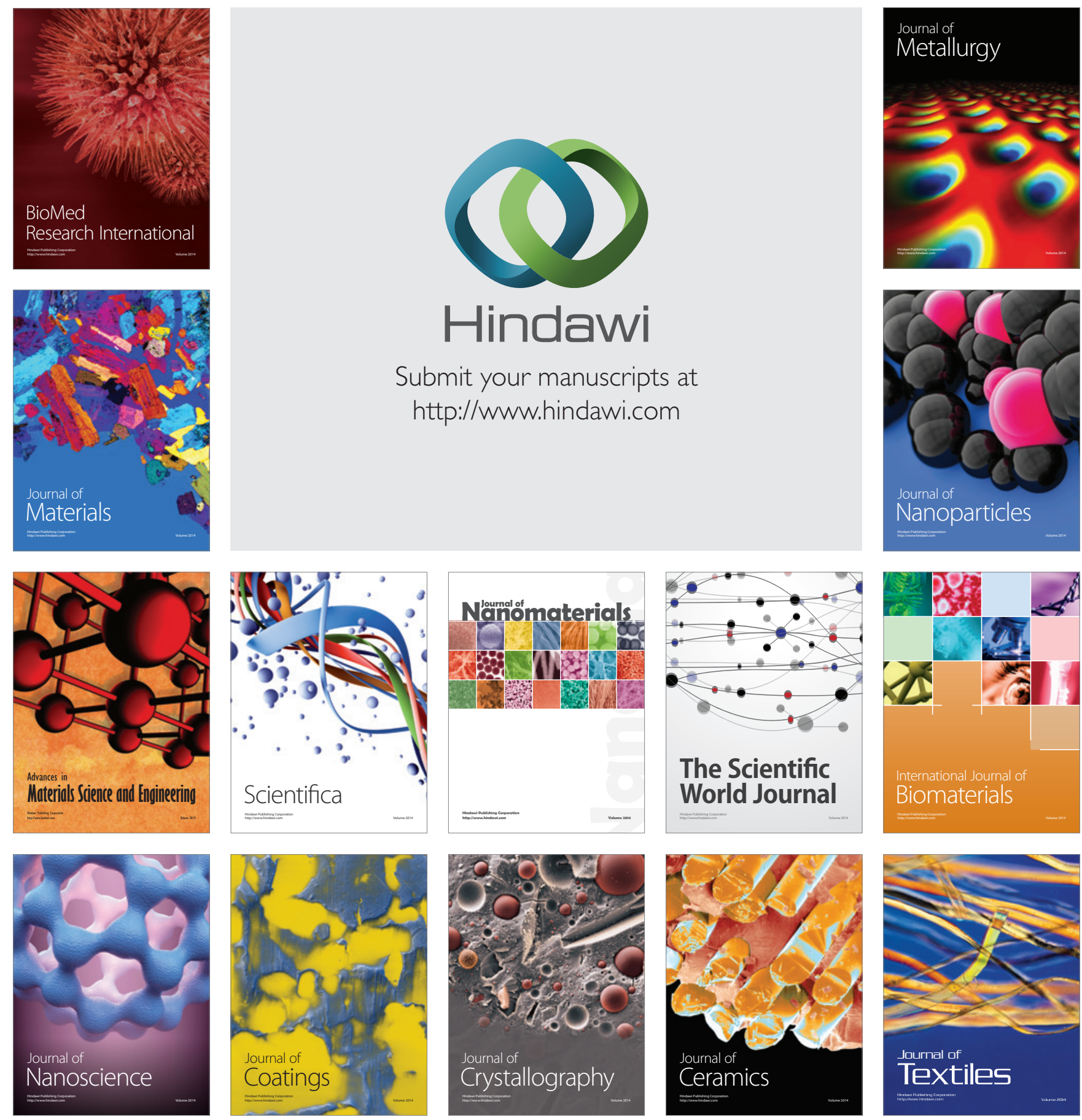